\title{
The Relationship between Hematoma Iron Content and Perihematoma Edema: An MRI Study
}

\author{
Min Lou ${ }^{a}$ Kathrin Lieb ${ }^{b}$ Magdy Selim ${ }^{b}$ \\ aSecond Affiliated Hospital of Zhejiang University, School of Medicine, Hangzhou, China; \\ ${ }^{b}$ Beth Israel Deaconess Medical Center, Harvard Medical School, Boston, Mass., USA
}

\section{Key Words}

Iron - Edema - Hematoma - Intracerebral hemorrhage •

Magnetic resonance imaging

\begin{abstract}
Background: Iron neurotoxicity has been linked to delayed neuronal injury and edema formation after intracerebral hemorrhage $(\mathrm{ICH})$. We have previously shown that serum ferritin, an indicator of body iron load, correlates with the relative perihematoma edema volume (RPHEV) on days 3-4 after ICH. We undertook this study to directly examine the relationship between in vivo brain and hematoma iron content, measured by MRI, and RPHEV. Methods: We retrospectively reviewed prospectively collected clinical and laboratory data from 36 consecutive patients with acute spontaneous lobar ICH who had MRI performed within 2-4 days of $\mathrm{ICH}$ onset. We measured hematoma and edema volumes, and the signal intensity on $\mathrm{T}_{2}$-weighted images $\left(\mathrm{T}_{2} \mathrm{SI}\right)$, as an estimate of iron content, in the hematoma and contralateral globus pallidus (GP). We calculated the RPHEV and $\mathrm{T}_{2} \mathrm{SI}$ in the hematoma and GP, relative to $\mathrm{T}_{2} \mathrm{SI}$ in the frontal deep white matter which contains negligible iron, to estimate the hematoma and brain iron load. We used Spearman correlation coefficient to determine the association of relative $\mathrm{T}_{2} \mathrm{SI}$ of the hematoma and GP with RPHEV. Results: We found a significant inverse correlation between the relative
\end{abstract}

$\mathrm{T}_{2} \mathrm{~S}$ in the hematoma $(r=-0.75, p<0.001)$ and to a lesser extent in the GP $(r=-0.34, p=0.04)$ and the RPHEV. Conclusions: Our findings suggest that in vivo brain and hematoma iron content, as measured by MRI, is linked to perihematoma edema after $\mathrm{ICH}$, and provide further support to existing preclinical evidence linking iron-mediated toxicity to delayed neuronal injury after $\mathrm{ICH}$.

Copyright $\odot 2009$ S. Karger AG, Basel

\section{Introduction}

Studies in animal models of intracerebral hemorrhage (ICH) show that hemoglobin and its degradation products, particularly iron, are neurotoxic and are causally related to delayed neuronal injury and edema formation after ICH [1-4]. Iron can promote oxidative damage by catalyzing hydroxyl radical formation and lipid peroxidation $[2,4]$. In a recent study, we observed a significant positive correlation between serum ferritin and relative perihematoma edema volume (RPHEV) on days 3-4 in patients with spontaneous ICH [5]. We used serum ferritin, which is a reliable indicator of body iron load [6], as an indirect measure of iron availability in the erythrocytes and the area of ICH since we could not directly assess iron content in the vicinity of the hemorrhagic brain tissue.

\section{KARGER}

(ㅇ) 2009 S. Karger AG, Basel

Fax +41613061234 E-Mail karger@karger.ch www.karger.com www.karger.com/ced
Magdy Selim, MD, PhD

Department of Neurology, Stroke Division, Beth Israel Deaconess Medical Center

330 Brookline Avenue, Palmer 127

Boston, MA 02215 (USA)

Tel. +1 617632 8913, Fax +1 617632 8920, E-Mail mselim@bidmc.harvard.edu 
Fig. 1. Representative pictures of hematoma and perihematoma edema measurements. a ROIs drawn around the perimeters of the perihematoma edema and hematoma. b ROIs drawn on the frontal white matter and GP.

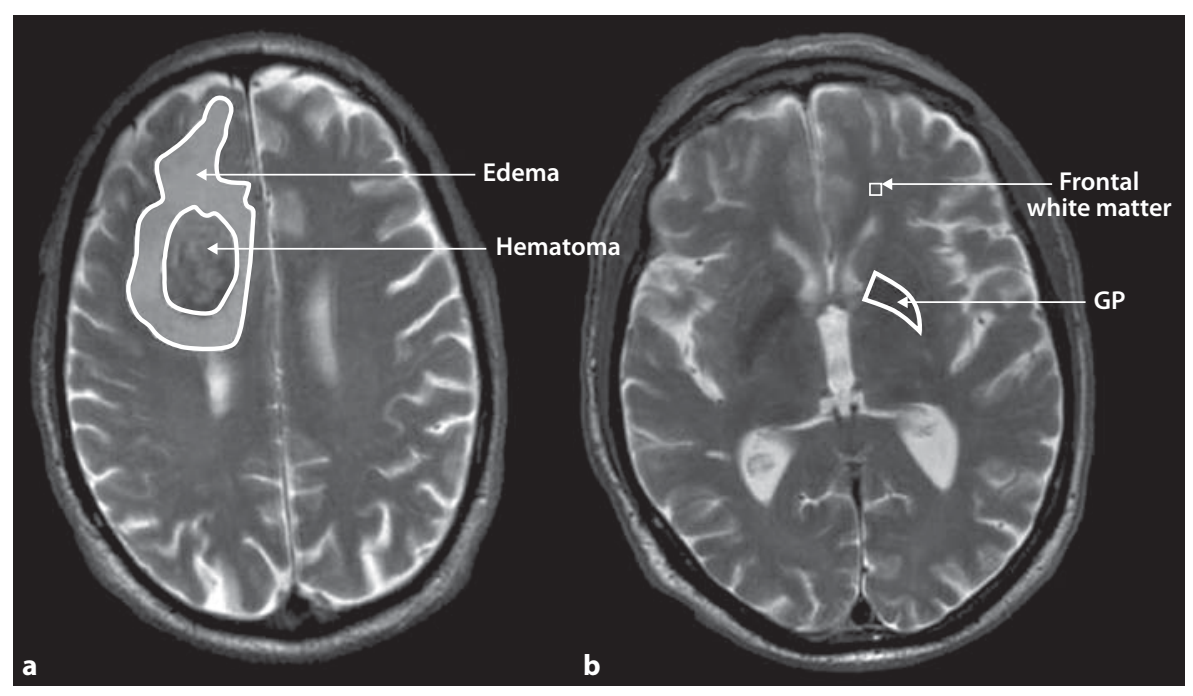

Iron deposition in the brain can be detected with magnetic resonance imaging (MRI) [7-11]. The iron atoms form ferric oxyhydroxide particles, which shorten the relaxation times on $\mathrm{T}_{2}$-weighted images resulting in darker (hypointense) signal in brain regions with higher iron content [7]. Signal intensity on $\mathrm{T}_{2}$-weighted images $\left(\mathrm{T}_{2} \mathrm{SI}\right)$ has been used to reflect the regional iron content of brain tissue in normal aging and neurodegenerative conditions associated with pathological disturbance of brain iron homeostasis [8-11]. Therefore, we employed MRI to assess in vivo brain iron in the current study to directly examine the relationship between brain and hematoma iron load and RPHEV in patients with acute spontaneous $\mathrm{ICH}$.

\section{Methods}

\section{Patient Selection and Data Collection}

We retrospectively reviewed our prospectively collected stroke database for consecutive patients with ICH admitted to our service during an 18-month period from 2006 to 2008 . We identified patients with spontaneous lobar ICH who had a brain MRI performed within 2-4 days of ICH symptom onset. Patients with secondary causes of ICH, such as anticoagulant use, underlying aneurysm vascular malformation, or tumor, head trauma, or hemorrhagic transformation of ischemic infarcts were excluded. In addition, we excluded patients with initial or subsequent intraventricular and infratentorial hemorrhages and hemorrhage in the basal ganglia to avoid technical flaws regarding accurate edema volume measurements and the effect of iron deposit in basal ganglia on signal intensity of deep hematoma in these situations. We also excluded patients who received antiedema treatments, or underwent surgical intervention during hospitalization before having the MRI scan. We retrieved demographic, clinical, laboratory, and radiological data, and examined the admission CT scans of eligible patients to confirm that none had evidence of calcification in the globus pallidus (GP) which could result in $\mathrm{T}_{2} \mathrm{SI}$ shortening on MRI.

\section{Radiological Measurements}

All MRI studies were performed on a 1.5-tesla MR whole-body scanner with echo-planar imaging capability, using a standardized imaging protocol including $\mathrm{T}_{2}$-weighted images. We measured the hematoma and edema volumes, and the signal intensity in the region of the hematoma and the contralateral GP and frontal lobe white matter on $T_{2}$ images off-line using the National Institute of Health imaging processing software. We measured the signal intensity in the contralateral GP and frontal white matter in order to avoid the confounding effects of ICH-associated edema in the ipsilateral hemisphere on $\mathrm{T}_{2} \mathrm{SI}$. A single evaluator (M.L.) experienced in the interpretation of MRI images analyzed all scans to conduct all measurements. Definition of the hematoma and perihematoma edema was assisted by adjustment of the contrast and threshold value until the hematoma consisting of a dark rim and bulk on $\mathrm{T}_{2}$ images could be well separated from the surrounding hyperintense edematous brain tissue in terms of appropriate high- and low-attenuation zones [12]. The examiner then manually drew regions of interest (ROIs) by tracing the perimeters of the hematoma and perihematoma edema in each slice throughout the hemorrhagic lesion (fig. 1a). The traced ROIs in contiguous voxels were then summed up after adjusting for the slice thickness to yield a hematoma volume and an absolute edema volume (the volume of the hematoma and surrounding edema) (fig. 1a). To control for hematoma volume, we subtracted the hematoma volume from that of the absolute edema, and divided the product by the hematoma volume, to express the perihematoma edema volume as a ratio of the associated hematoma volume (RPHEV).

The observer (M.L.) drew the ROIs on an initial subset of 10 patients twice, at an interval of 6 months apart. The intraobserv- 
Table 1. Summary of hematoma and edema (absolute and relative) volumes

\begin{tabular}{|c|c|c|c|}
\hline Volume & $\begin{array}{l}\text { Hematoma } \\
\mathrm{cm}^{3}\end{array}$ & $\begin{array}{l}\text { Absolute } \\
\text { edema, } \mathrm{cm}^{3}\end{array}$ & $\begin{array}{l}\text { Relative } \\
\text { edema }\end{array}$ \\
\hline Median & 16.90 & 20.57 & 1.43 \\
\hline Range & $0.77-96.42$ & $1.49-89.48$ & $0.53-3.02$ \\
\hline Mean $\pm S D$ & $23.79 \pm 22.43$ & $26.92 \pm 20.56$ & $1.49 \pm 0.72$ \\
\hline
\end{tabular}

Table 2. Summary of relative $\mathrm{T}_{2} \mathrm{SI}$ in the hematoma and contralateral GP

\begin{tabular}{lll}
\hline & $\mathrm{T}_{2} \mathrm{SI}$ of hematoma & $\mathrm{T}_{2} \mathrm{SI}$ of GP \\
\hline Median (range) & $1.90(0.80-2.70)$ & $0.61(0.49-1.10)$ \\
Mean \pm SD & $1.42 \pm 0.47$ & $0.91 \pm 0.13$ \\
\hline
\end{tabular}

er test/retest agreement was $r=0.95$ for absolute edema volume, $\mathrm{r}=0.98$ for hematoma volume measurements. Another investigator (K.L.) independently drew the ROIs on the same subset of 10 patients. The interobserver agreement was $r=0.91$ for absolute edema volume and $\mathrm{r}=0.98$ for hematoma volume measurements. Because the intra- and interobserver reliability measures were high, only one measurement by 1 observer (M.L.) was used for the remainder of the ROIs.

ROIs were also manually drawn in the GP and frontal white matter, contralateral to the hematoma (fig. 1b). The GP was chosen because it has the highest iron content in the brain and is easily visible on $\mathrm{T}_{2}$ images [13-15]. The frontal white matter was selected, as a control, because it contains negligible amounts of iron [13-15]. The $\mathrm{T}_{2} \mathrm{SI}$ of hematoma and GP ROIs in each voxel was automatically measured as a gray scale and summed up to calculate the average $\mathrm{T}_{2} \mathrm{SI}$ in each of these regions. A lower $\mathrm{T}_{2} \mathrm{SI}$ value represents more $\mathrm{T}_{2}$ shortening (darker signal), and presumably reflects higher iron content. Three measurements were made on the frontal white matter with a $3-\mathrm{mm}^{2}$ square ROI and then averaged to obtain a final value, which was used as an internal reference. We then calculated the relative $\mathrm{T}_{2} \mathrm{SI}$ of the hematoma and GP after dividing the mean $\mathrm{T}_{2} \mathrm{SI}$ within each region by that of the frontal white matter to derive MRI estimates of relative iron content in the hematoma and GP. A lower ratio between mean regional hematoma or GP and white matter $\mathrm{T}_{2} \mathrm{SI}$ indicated a lower $\mathrm{T}_{2} \mathrm{SI}$, presumably related to higher iron content within these regions.

\section{Statistical Analysis}

We used the Spearman correlation coefficient to determine the presence or absence of a correlation between the relative $\mathrm{T}_{2} \mathrm{SI}$ of the hematoma, as a measure of hematoma iron load, and GP, as a measure of brain iron load, and RPHEV. Statistical significance was set at a probability value of $\leq 0.05$.

\section{Results}

A total of 36 subjects who met all of our inclusion and none of our exclusion criteria were included in the present analysis. Sixty-one percent were men, and 39\% women. Mean age was $74.6 \pm 16.1$ years (range: $28-93$ ). Mean onset-to-imaging time for the MRI scan was $2.0 \pm 1.0$ days. Thirteen patients (36\%) were discharged home, 22 (61\%) to extended-care facilities or rehabilitation, and 1 (3\%) died during hospitalization. None of the patients had conditions that could affect the $\mathrm{T}_{2} \mathrm{SI}$ on MRI, such as severe anemia, Parkinson's disease, Hunting ton's disease, schizophrenia, demyelinating disease, or visible calcification of the basal ganglia on admission CT scan.

Table 1 lists the hematoma and perihematoma edema volumes on $\mathrm{T}_{2}$-weighted MRI. The mean $\mathrm{T}_{2} \mathrm{SI}$ in the subcortical frontal white matter was $61 \pm 18$. Table 2 lists the $\mathrm{T}_{2} \mathrm{SI}$ in the hematoma region and contralateral GP relative to the frontal subcortical white matter. The ratio for $\mathrm{T}_{2} \mathrm{SI}$ between the GP and white matter ranged from 0.49 to 1.1 and that between the hematoma and white matter from 0.80 to 2.7 , reflecting the large variability in brain and hematoma iron content between the subjects. Figure 2 depicts the correlation between the relative $\mathrm{T}_{2} \mathrm{SI}$ in the hematoma region and the RPHEV. As it shows, there was a significant inverse correlation between the relative $\mathrm{T}_{2} \mathrm{SI}$ of the hematoma and the RPHEV $(\mathrm{r}=-0.75, \mathrm{p}<$ 0.001). Similarly, figure 3 shows that there was also an inverse correlation, albeit less robust, between the relative $\mathrm{T}_{2} \mathrm{SI}$ of the GP and the RPHEV $(\mathrm{r}=-0.34, \mathrm{p}=0.04)$. We found no correlation between the $\mathrm{T}_{2} \mathrm{SI}$ in the frontal white matter and RPHEV $(r=0.219, \mathrm{p}=0.199)$, or between the hematoma volume and $\mathrm{T}_{2}$ SI within it $(\mathrm{r}=0.303$, $\mathrm{p}=0.073)$.

\section{Discussion}

We found significant inverse correlations between the relative $\mathrm{T}_{2} \mathrm{SI}$ in the hematoma region and GP, and the RPHEV in this cohort of patients with acute spontaneous lobar ICH. Our results indicate that higher iron content in the hematoma region and to a lesser extent in the GP correlate with perihematoma edema volume after ICH. These findings lend further support to the important role of iron-mediated neurotoxicity after ICH.

Evidence linking iron to neuronal injury and perihematoma edema formation derives mostly from studies in experimental models of ICH [1-4]. Several studies have shown that hemoglobin and its degradation products, in 


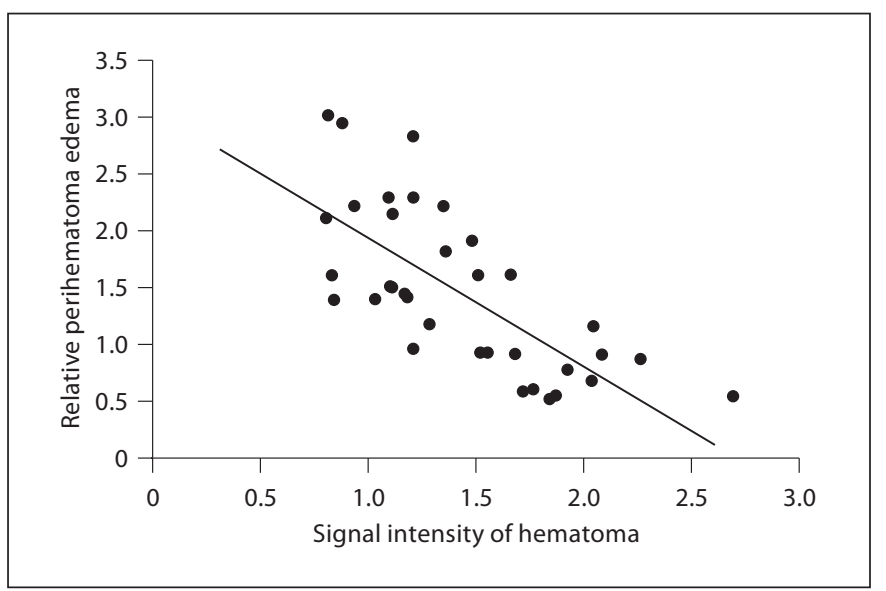

Fig. 2. Scatter plot showing the correlation between RPHEV and relative $\mathrm{T}_{2} \mathrm{SI}$ of hematoma $(\mathrm{r}=-0.75, \mathrm{p}<0.001, \mathrm{n}=36)$.

particular iron, resulting from erythrocyte lysis after ICH exert neurotoxic effects and contribute to the development and progression of brain edema following ICH and that treatment with iron chelators can reduce these effects $[1-4,16,17]$. There have not been many clinical studies examining the status and role of iron in the brain. We recently observed a positive correlation between serum ferritin levels on admission and the RPHEV on days 3-4 after ICH onset, suggesting that increased body iron load is associated with edema progression after ICH [5]. We undertook the current study in order to further examine the relationship between in vivo iron load in the brain and the hematoma and RPHEV after ICH.

MRI is a valuable assessment tool with its unique specificity to hemoglobin degradation products and iron. The specific appearance of hemoglobin and blood degradation products on MRI sequences has been extensively described in the literature $[12,18,19]$. Rapid deoxygenation of blood occurs at the border of the hematoma, which leads to a transition from the diamagnetic oxyhemoglobin to the paramagnetic deoxyhemoglobin. This creates a 'boundary effect' leading to signal loss (hypointensity) at the periphery of the hematoma and separating the hematoma well from the surrounding hyperintense edematous tissue [12]. The hypointensity of the hematoma on $\mathrm{T}_{2}$ images reflects the paramagnetic iron-containing hemoglobin degradation products, which can be released from the erythrocytes in the area of ICH at the early stage $[2,3]$. The iron molecules in the brain tissue and in the hemoglobin-degradation products within the hematoma region have unpaired elec-

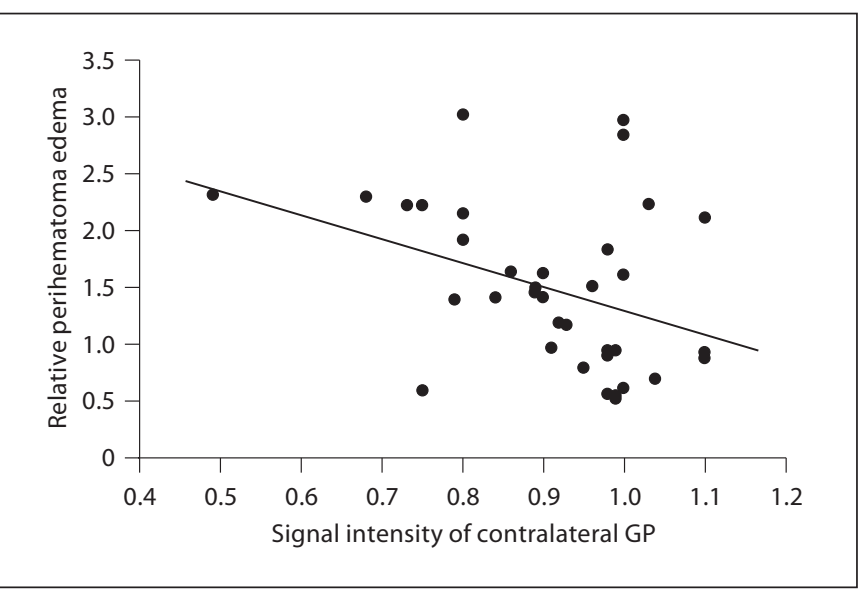

Fig. 3. Scatter plot showing the correlation between RPHEV and $\mathrm{T}_{2} \mathrm{SI}$ of contralateral GP $(\mathrm{r}=-0.34, \mathrm{p}=0.04, \mathrm{n}=36)$.

trons, i.e. paramagnetic properties, which shorten the $T_{2}$ relaxation time and produce visible hypointensity on $\mathrm{T}_{2^{-}}$ weighted images caused by dephasing of water protons as they diffuse through the local magnetic field inhomogeneities created by the presence of the iron particles $[15$, $18-20] . \mathrm{T}_{2} \mathrm{SI}$ is influenced by regional iron content. Several investigators have reported a correlation between $\mathrm{T}_{2}$ shortening and regional brain tissue iron concentration, using the mean iron concentration of various brain regions and structures from postmortem and autopsy studies as a reference to validate their findings $[13,15$, 21-23]. Accordingly, we used MRI $\mathrm{T}_{2} \mathrm{SI}$ as a surrogate measure to estimate in vivo the regional iron content of the hematoma in this study. Postmortem studies indicate that the basal ganglia, in particular GP, contain the highest levels of iron in the brain [13-15]. Therefore, we also examined the $\mathrm{T}_{2} \mathrm{SI}$ of the GP as an in vivo indicator of the total brain iron load.

The iron content in the brain increases gradually with age, up to about 50 years $[8,13,23]$. We found no significant correlations between age and relative $\mathrm{T}_{2} \mathrm{SI}$ in the GP, or age and RPHEV in the current study (unreported data). This is likely attributed to the fact that the vast majority of our patients were older than 60 years of age. Our finding of a modest correlation between relative $\mathrm{T}_{2} \mathrm{SI}$ in the GP, as an indicator of brain iron load [13-15], and RPHEV implies that brain iron status may be an important determinant of secondary neuronal injury and edema formation after ICH. However, it is possible that increased iron load in the GP could be secondary to ICH itself. Further studies are required to exam- 
ine the relationship between baseline brain iron status and RPHEV.

Our findings indicate that the use of MRI to estimate in vivo regional iron content is feasible in ICH patients and that MRI can be potentially used as a tool to assess or guide interventions aimed at decreasing brain iron levels in future studies.

There are limitations to our study that are largely imposed by its retrospective nature. First, independent histopathological confirmation of $\mathrm{T}_{2} \mathrm{SI}$ estimates of tissue iron was not feasible. However, previous histological and postmortem studies have reported a correlation between $\mathrm{T}_{2} \mathrm{SI}$ and regional brain tissue iron concentration $[13,15$, 21-23]. Second, the $\mathrm{T}_{2} \mathrm{SI}$ of MR images depends on a variety of factors other than the presence of paramagnetic iron particles, such as regional tissue characteristics [14]. Other techniques, such as MRI field-dependent $R_{2}$ increase, to quantify brain iron levels may be more specific and superior to the semiquantitative methodology using $\mathrm{T}_{2} \mathrm{SI}$ employed in this study [24]. However, this technique requires the use of two different field-strength MRI instruments. Third, we could not separately analyze the specific contribution of various forms of ironcontaining degradation products to the $\mathrm{T}_{2} \mathrm{SI}$. It is unlikely that methemoglobin or hemosiderin significantly contributed to the $\mathrm{T}_{2} \mathrm{SI}$ of the hematoma in the current study since MRI was performed during the acute phase within 2-4 days of ICH onset [18]. Lastly, we could not measure the temporal evolution of $\mathrm{T}_{2} \mathrm{SI}$ and perihematoma edema at different time intervals after ICH onset. It is therefore unknown if the $\mathrm{T}_{2} \mathrm{SI}$ of the hematoma in this early period bears any relationship to the evolution of perihematomal edema beyond the acute phase of $\mathrm{ICH}$. The above limitations need to be considered when interpreting our findings.

In conclusion, we found that the relative degree of $\mathrm{T}_{2}$ shortening (hypointensity) in the hematoma region and GP correlates with RPHEV, suggesting that iron content in the brain and hematoma influences the extent of the surrounding edema. Future prospective studies using more advanced MRI techniques are warranted to confirm our findings.

\section{Acknowledgments}

Dr. Lou is supported by a Zhejiang University Academic Stars Research Fellowship and grants from the National Natural Science Foundation of China (30500175); Dr. Selim is supported in part by grants from the NINDS (1R01-NS 057127-01A1) and NIH (5R01-HL46690-14).

\section{References}

1 Hua Y, Keep RF, Hoff JT, Xi G: Brain injury after intracerebral hemorrhage: the role of thrombin and iron. Stroke 2007;38(suppl 2):759-762.

2 Nakamura T, Keep RF, Hua Y, Nagao S, Hoff JT, Xi G: Iron-induced oxidative brain injury after experimental intracerebral hemorrhage. Acta Neurochir Suppl 2006;96:194198.

3 Wagner KR, Sharp FR, Ardizzone TD, Lu A, Clark JF: Heme and iron metabolism: role in cerebral hemorrhage. J Cereb Blood Flow Metab 2003;23:629-652.

4 Wu J, Hua Y, Keep RF, Schallert T, Hoff JT, $\mathrm{Xi}$ G: Oxidative brain injury from extravasated erythrocytes after intracerebral hemorrhage. Brain Res 2002;953:45-52.

5 Mehdiratta M, Kumar S, Hackney D, Schlaug G, Selim M: Association between serum ferritin level and perihematoma edema volume in patients with spontaneous intracerebral hemorrhage. Stroke 2008;39:1165-1170.

$\checkmark 6$ Cook JD: Defining optimal body iron. Proc Nutr Soc 1999;58:489-495.
7 Bartzokis G, Sultzer D, Cummings J, Holt L, Hance D, Henderson V, Mintz J: In vivo evaluation of brain iron in Alzheimer disease using magnetic resonance imaging. Arch Gen Psychiatry 2000;57:47-53.

8 Siemonsen S, Finsterbusch J, Matschke J, Lorenzen A, Ding XQ, Fiehler J: Age-dependent normal values of $\mathrm{T}^{*}$ and $\mathrm{T} 2$ ' in brain parenchyma. AJNR Am J Neuroradiol 2008; 29:950-955.

-9 Kosta P, Argyropoulou MI, Markoula S, Konitsiotis S: MRI evaluation of the basal ganglia size and iron content in patients with Parkinson's disease. J Neurol 2006;253:2632.

10 Godau J, Klose U, Di Santo A, Schweitzer K, Berg D: Multiregional brain iron deficiency in restless legs syndrome. Mov Disord 2008; 23:1184-1187.

-11 McNeill A, Birchall D, Hayflick SJ, Gregory A, Schenk JF, Zimmerman EA, Shang H, Miyajima $\mathrm{H}$, Chinnery PF: T2* and FSE MRI distinguishes four subtypes of neurodegeneration with brain iron accumulation. Neurology 2008;70:1614-1619.
12 Alemany Ripoll M, Stenborg A, Sonninen P, Terent A, Raininko R: Detection and appearance of intraparenchymal haematomas of the brain at $1.5 \mathrm{~T}$ with spin-echo, FLAIR and GE sequences: poor relationship to the age of the haematoma. Neuroradiology 2004;46: 435-443.

13 Hallgren B, Sourander P: The effect of age on the non-hemin iron in the human brain. J Neurochem 1958;3:41-51.

14 Chen JC, Hardy PA, Kucharczyk W, Clauberg M, Joshi JG, Vourlas A, Dhar M, Henkelman RM: MR of human postmortem brain tissue: correlative study between T2 and assays of iron and ferritin in Parkinson and Huntington disease. AJNR Am J Neuroradiol 1993;14:275-281.

15 Bizzi A, Brooks RA, Brunetti A, Hill JM, Alger JR, Miletich RS, Francavilla TL, Di Chiro G: Role of iron and ferritin in MR imaging of the brain: a study in primates at different field strengths. Radiology 1990;177:59-65.

16 XiG, Keep RF, Hoff JT: Erythrocytes and delayed brain edema formation following intracerebral hemorrhage in rats. J Neurosurg 1998;89:991-996. 
17 Nakamura T, Keep R, Hua Y, Schallert T, Hoff J, Xi G: Deferoxamine-induced attenuation of brain edema and neurological deficits in a rat model of intracerebral hemorrhage. J Neurosurg 2004;100:672-678.

18 Bradley WG Jr: MR appearance of hemorrhage in the brain. Radiology 1993;189:1526.

-19 Atlas SW, Thulborn KR: MR detection of hyperacute parenchymal hemorrhage of the brain. AJNR Am J Neuroradiol 1998; 19: 1471-1477.
0 Brooks R, Vymazal A, Bulte J, Baumgarner C, Tran V: Comparison of T2 relaxation in blood, brain and ferritin. J Magn Reson Imaging 1995;5:446-450.

-21 Ordidge R, Gorell J, Deniau J, Knight R, Helpern J: Assessment of relative brain iron concentration using T2-weighted and $\mathrm{T}^{*}$ weighted MRI at 3 Tesla. Magn Reson Med 1994;32:335-341.

22 Imon Y, Yamaguchi S, Katayama S, Oka M, Murata Y, Kajima T, Yamamura Y, Nakamura S: A decrease in cerebral cortex intensity on T2-weighted with ageing images of normal subjects. Neuroradiology 1998;40:7680
23 Bartzokis G, Mintz J, Sultzer D, Marx P, Herzberg JS, Phelan CK, Marder SR: In vivo MR evaluation of age-related increases in brain iron. AJNR Am J Neuroradiol 1994;15: 1129-1138.

24 Bartzokis G, Tishler T, Shin I, Lu P, Cummings J: Brain ferritin iron as a risk factor for age at onset in neurodegenerative diseases. Ann NY Acad Sci 2004;1012:224-236. 